\title{
Note on BEC in Nonextensive Statistical Mechanics
}

\author{
Kwok Sau $\mathrm{Fa}^{1}$ and E. K. Lenzi ${ }^{2}$ \\ ${ }^{1}$ Departamento de Física, Universidade Estadual de Maringá, \\ Av. Colombo 5790, 87020-900 Maringá-PR, Brazil \\ ${ }^{2}$ Centro Brasileiro de Pesquisas Físicas, R. Xavier Sigaud 150, \\ 22290-180 Rio de Janeiro, Brazil
}

Received on 17 October, 2000. Revised version received on 8 January, 2001

\begin{abstract}
The generalized Bose-Einstein distribution, within the dilute gas assumption, in the nonextensive Tsallis statistics is worked without approximation for the Bose-Einstein condensation (BEC). The results obtained are compared with the recent results presented in Int. J. Mod. Phys. B 14, 405 (2000) by L. Salasnich. Furthermore, in order to promote a complete analysis for the BEC in the nonextensive scenario we also find exact expression within the normalized constraints in a harmonic trap.
\end{abstract}

\section{Introduction}

In this last decade, we have witnessed a growing interest in the Tsallis statistics $[1,2]$. The starting point of Tsallis statistics is based on the nonextensive entropy $S_{q}=k\left(1-\sum_{i=1}^{W} p_{i}^{q}\right) /(q-1)$, where $k$ is a positive constant and $q \in R$. In the limit $q \rightarrow 1$ the usual BoltzmanGibbs entropy is recovered. It has been applied in many situations such as, Lévy-type anomalous superdiffusion [3], Euler turbulence [4], self-gravitating and correlated systems [5], anomalous relaxation through electronphonon interaction [6], ferrofluid-like systems [7], and among others [8]. In particular, the Bose-Einstein and Fermi-Dirac distributions have been extensively analyzed in the Tsallis framework [6,9-16]. Further, we can mention the specific heat obtained for ${ }^{4} \mathrm{He}$ [17] which is in agreement with the experimental results reported in [18]. For the most of these cases the analyses involving these generalized Bose-Eisntein and Fermi-Dirac distribution have been restricted for free systems, i.e., the interaction between the particles is absent. However, important questions about the complete solution for the Bose-Einstein distribution and its implications for the thermodynamics of these physical systems are still present in this formalism. In this context, it may not be out of place to mention here the generalized BoseEinstein distribution in the dilute gas assumption [13], given by,

$$
\langle n(\epsilon)\rangle_{q}=\frac{1}{[1+\beta(q-1)(\epsilon-\mu)]^{1 /(q-1)}-1},
$$

and its expansion at first order in $(q-1)$

$$
\langle n(\epsilon)\rangle_{q}=\frac{1}{e^{\beta(\epsilon-\mu)}-1}+\frac{1}{2}(q-1) \frac{\beta^{2}(\epsilon-\mu)^{2} e^{\beta(\epsilon-\mu)}}{\left[e^{\beta(\epsilon-\mu)}-1\right]^{2}} .
$$

The equation (2) has been applied widely in the literature [6, 12, 13, 14, 15] (see [8] and references therein) for obtaining thermodynamical quantities that emerge from it. In this way, a complete solution involving Eq.(1) can be useful for quantitative and qualitative analysis of systems in the nonextensive context. Furthermore, the development based on Eq.(1) can also be useful in the description of the systems with (multi)fractal structure (similar situation in metals [19] has been analyzed in [16]), systems with longtime memories, and among others. Another important point is about the constraints and the relation between the Lagrange parameter $\beta$ and temperature in the formulations of Tsallis statistics[2].

Thus, we propose, in this work to find the complete solution for the BEC transition temperature considering $D$ dimension and $q>0$, within the dilute gas assumption. Then, we compare it with the approximate results presented in the literature, and in particular the Salasnich's work[12], by varying the values of $q$ and $D$, and as a consequence to check the validity of the approximation (2). In addition, we show that the results obtained by using Eq.(2) are not accurate for most situations when compared with our ones. Also, to promote a future discussion involving the constraints, Lagrange parameters, temperature and experimental data, we ob- 
tain the Bose-Einstein distribution by taking into account the normalized constraints[2].

In order to facilitate our analysis, let us recall some results evaluated in [12] with Eq.(2). The quantities evaluated in [12] are: the BEC transition tempera- ture, the condensed fraction and the energy per particle to the homogeneous gas, the gas in a harmonic trap and the relativistic homogeneous gas in D-dimensional space. For non-interacting bosons, the total number of particle is evaluated by the following expression

$$
N=\int_{0}^{\infty} \mathrm{d} \epsilon \rho(\epsilon)\langle n(\epsilon)\rangle_{q},
$$

where $\rho(\epsilon)$ is the density of states. In a D-dimensional box of volume $V$, the densities of states for homogeneous gas and gas in harmonic trap are given respectively by $\rho(\epsilon)=\left\{(V / \epsilon)\left[m \epsilon /\left(2 \pi \hbar^{2}\right)\right]^{D / 2}\right\} / \Gamma(D / 2)$ and $\rho(\epsilon)=$ $\left\{[\epsilon /(\hbar \bar{\omega})]^{D}\right\} /(\Gamma(D) \epsilon)$, where $\bar{\omega}$ is the geometric average of the trap frequencies and $\Gamma(x)$ is the gamma function.

To calculate the BEC transition temperature $T_{q}$, with $\mu=0$, we substitute Eq.(2) and $\rho(\epsilon)$ into (3), and we obtain

$$
k T_{q}=\left(\frac{2 \pi \hbar^{2}}{m}\right) \frac{(N / V)^{2 / D} /[\zeta(D / 2)]^{2 / D}}{\left[1+\frac{q-1}{2} \frac{\Gamma(D / 2+2) \zeta(D / 2+1)}{\Gamma(D / 2) \zeta(D / 2)}\right]^{2 / D}},
$$

for the homogeneous gas, and

$$
k T_{q}=\frac{\hbar \bar{\omega} N^{1 / D}}{[\zeta(D)]^{1 / D}\left[1+\frac{q-1}{2} \frac{\Gamma(D+2) \zeta(D+1)}{\Gamma(D) \zeta(D)}\right]^{1 / D}},
$$

for a gas in a harmonic trap. $\zeta(x)$ is the Riemann $\zeta$-function. The energy is calculated by the following expression

$$
E=\int_{0}^{\infty} \mathrm{d} \epsilon \epsilon \rho(\epsilon)\langle n(\epsilon)\rangle_{q}
$$

The results are

$$
\frac{E}{k T_{q}}=\frac{V D}{2}\left(\frac{m k T_{q}}{2 \pi \hbar^{2}}\right)^{D / 2} \zeta(D / 2+1)\left[1+\frac{q-1}{2} \frac{\Gamma(D / 2+3) \zeta(D / 2+2)}{\Gamma(D / 2+1) \zeta(D / 2+1)}\right],
$$

for the homogeneous gas, and

$$
\frac{E}{k T_{q}}=\left(\frac{k T_{q}}{\hbar \bar{\omega}}\right)^{D} D \zeta(D+1)\left[1+\frac{q-1}{2} \frac{\Gamma(D+3) \zeta(D+2)}{\Gamma(D+1) \zeta(D+1)}\right],
$$

for a gas in a harmonic trap.

For the relativistic gas, the total number of particles is not conserved due to the production of antiparticles, but the difference between the number $N$ of particles and the number $N$ of antiparticles is conserved, i.e., $Q=N-\bar{N}=$ $\int_{0}^{\infty} \mathrm{d} \epsilon \rho(\epsilon)\left[\langle n(\epsilon)\rangle_{q}-\langle\bar{n}(\epsilon)\rangle_{q}\right]$, where $\langle\bar{n}(\epsilon)\rangle_{q}$ is obtained from $\langle n(\epsilon)\rangle_{q}$ by replacing $\mu \rightarrow-\mu$. The density of states for the relativistic gas is given by $\left.\rho(\epsilon)=\left(V 2 \pi^{D / 2}\right) /\left(2 \pi \hbar^{2}\right)^{D} \Gamma(D / 2)\right) \epsilon\left(\epsilon^{2}-m^{2} c^{4}\right)^{(D-2) / 2}$. For ultrarelativistic region $k T>>m c^{2}$, the critical temperature at which BEC occurs corresponds to $|\mu|=m c^{2}$. Expanding $Q$ at first order in $\mu$ yields

$$
k T_{q}=\left[\frac{(2 \pi \hbar c)^{D} \Gamma(D / 2)|Q|}{4 V \pi^{D / 2} \Gamma(D) \zeta(D-1) m c^{2}}\right]^{1 /(D-1)}\left[1+\frac{q-1}{2} \frac{(D-1) D \zeta(D)}{\zeta(D-1)}\right]^{-1 /(D-1)} .
$$

The Eqs.(4), (5), (6), (7) and (9) are the results obtained in [12], by using Eq.(2). Similar results for free particles were also obtained in [15]. Now, we calculate again the quantities above by using the distribution (1), instead of (2). To do so, we note that the distribution (1) can be written as a sum, i.e.,

$$
\begin{aligned}
\langle n(\epsilon)\rangle_{q} & =\frac{1}{[1+\beta(q-1)(\epsilon-\mu)]^{\frac{1}{q-1}}-1} \\
& =\sum_{n=1}^{\infty}[1+\beta(q-1)(\epsilon-\mu)]^{-n /(q-1)} .
\end{aligned}
$$


Substituting $\rho(\epsilon)$ and Eq.(10) into (3) and taking into account the cut-off [2] for $q<1$, we obtain the critical temperature as

$$
\frac{m k T_{q}}{2 \pi \hbar^{2}}=\left\{\begin{array}{lc}
{\left[\frac{V}{N} \sum_{n=1}^{\infty} \frac{(1-q)^{-D / 2} \Gamma\left(\frac{n}{1-q}+1\right)}{\Gamma\left(\frac{n}{1-q}+1+\frac{D}{2}\right)}\right]^{-\frac{2}{D}},} & 0<q<1 \\
{\left[\frac{V}{N} \sum_{n=1}^{\infty} \frac{(q-1)^{-D / 2} \Gamma\left(\frac{n}{q-1}-\frac{D}{2}\right)}{\Gamma\left(\frac{n}{q-1}\right)}\right]^{-\frac{2}{D}},} & q>1
\end{array}\right.
$$

for the homogeneous gas, and

$$
k T_{q}=(\hbar \bar{\omega}) N^{1 / D}\left[\sum_{n=1}^{\infty} \prod_{l=1}^{D} \frac{1}{n+(1-q) l}\right]^{-1 / D} \quad q>0
$$

for a gas in a harmonic trap. Note that Eq. (12) is valid for $(q-1)<1 / D$. In addition, the energy is also easily obtained from Eq.(6)

$$
\frac{E / V}{k T_{q}}=\left\{\begin{array}{cc}
\sum_{n=1}^{\infty} \frac{\left((1-q) m k T_{q}\right)^{D / 2+1} D \Gamma\left(\frac{n}{1-q}+1\right)}{2 m k T_{q}\left(2 \pi \hbar^{2}\right)^{D / 2} \Gamma\left(\frac{n}{1-q}+2+\frac{D}{2}\right)}, & 0<q<1 \\
\sum_{n=1}^{\infty} \frac{\left((q-1) m k T_{q}\right)^{D / 2+1} D \Gamma\left(\frac{n}{q-1}-\frac{D}{2}-1\right)}{2 m k T_{q}\left(2 \pi \hbar^{2}\right)^{D / 2} \Gamma\left(\frac{n}{q-1}\right)}, & q>1
\end{array}\right.
$$

for the homogeneous gas, and

$$
\frac{E}{k T_{q}}=D\left(\frac{k T_{q}}{\hbar \bar{\omega}}\right)^{D} \sum_{n=1}^{\infty} \prod_{l=1}^{D+1} \frac{1}{n+(1-q) l} \quad q>0
$$

for a gas in a harmonic trap. The Eq. (14) is valid for $(q-1)<1 /(1+D)$. We note that our results differ from those obtained by using the distribution (2). To see that closely, we quote $T_{q}$ with different values of $D$, for $q=1.1$ (see Table 1 ). We also plot the critical temperature in function of $q$. For homogeneous gas the convergence of the series of Eq. (11) is slow for $D=3$. For convenience, we plot $T_{q}$ in function of $q$, for $D=4$ (Fig. 1). For a gas in a harmonic trap, we plot $T_{q}$ in function of $q$, for $D=3$ (Fig. 2). As we can see the values quoted in Table 1, and the two figures (Figs.1 and 2) show clearly that the critical temperatures obtained by using (2) are not accurate when compared with our results. Even for a gas in a harmonic trap with small deviation in the nonextensive parameter $q=1.1$ and $D=3$, the divergence between our result and Salasnich's result [12] is remarkable. This is not surprise because the expansion made in (2), in the parameter $(q-1)$, has included the factor $\beta \epsilon$. This last factor is not necessarily small so the expansion of the exponential function at first order of $(q-1)$ cannot be good in general, especially when $|1-q|$ and $D$ are increased. We should also stress that the changes in the BEC transition temperature (for $q>1$ ), from the extensive Bose statistics, are greater than those obtained by using the distribution (2). For the relativistic gas, in the ultrarelativistic region $k T \gg m c^{2}$, the critical temperature by expanding $Q$ at first order in $\mu$ is given by

\begin{tabular}{llllllll}
\hline \hline$k T_{q} /\left(\hbar \bar{\omega} N^{1 / D}\right)$ & $D=3$ & $D=4$ & $D=5$ & $D=6$ & $D=7$ & $D=8$ & $D=9$ \\
\hline Salasnich & 0.8144 & 0.8288 & 0.8284 & 0.8266 & 0.8258 & 0.8261 & 0.8273 \\
our results & 0.7635 & 0.7342 & 0.6837 & 0.6262 & 0.5639 & 0.4954 & 0.4147 \\
\hline \hline
\end{tabular}

Table 1. Results for critical temperature of a gas in a harmonic trap system with $q=1.1$. The second row shows the results obtained by using the distribution (2) which are given by Eq. (5). While, the last row shows our results obtained by using the distribution (1) which are given by Eq. (12). 


$$
\frac{1}{k T_{q}}=\left\{\frac{4 V \pi^{D / 2} \Gamma(D) m c^{2}}{|Q|(2 \pi \hbar c)^{D} \Gamma(D / 2)} \sum_{n=1}^{\infty} \prod_{l=1}^{D-1} \frac{1}{n+(1-q) l}\right\}^{\frac{1}{D-1}} \quad q>0 .
$$

Eq.(15) also differs from that obtained by using the distribution (2). For example: for $q=1.1$ and $D=4$ we obtain $\left[\left(4 V \pi^{2} m c^{2}\right) /\left(|Q|(h c)^{4}\right)\right]^{1 / 3} k T_{q}=0.420181$ from Eq. (15) and $\left[\left(4 V \pi^{2} m c^{2}\right) /\left(|Q|(h c)^{4}\right)\right]^{1 / 3} k T_{q}=0.448173$ from Eq. (9).

For completeness, we show below $q$-mean value of particle number and internal energy in the normalized constraints [2] by taking into account $\mu=0$

$$
\begin{gathered}
\frac{\langle\hat{N}\rangle_{q}}{\langle\hat{N}\rangle_{1}}=\left(\frac{1+(1-q) \tilde{\beta} U_{q}}{(1-q) \tilde{\beta} / \beta_{c}^{(1)}}\right)^{D}\left\{\frac{\phi\left(D, \frac{1}{1-q}+D,\langle\hat{N}\rangle_{1}\left(\frac{1+(1-q) \tilde{\beta} U_{q}}{(1-q) \tilde{\beta} / \beta_{c}^{(1)}}\right)^{D} \frac{\zeta(D+1)}{\zeta(D)}\right)}{\phi\left(D, \frac{1}{1-q},\langle\hat{N}\rangle_{1}\left(\frac{1+(1-q) \tilde{\beta} U_{q}}{(1-q) \tilde{\beta} / \beta_{c}^{(1)}}\right)^{D} \frac{\zeta(D+1)}{\zeta(D)}\right)}\right\}, \\
\frac{U_{q} \beta_{c}^{(1)}}{D\langle\hat{N}\rangle_{1}}=\left(\frac{1+(1-q) \tilde{\beta} U_{q}}{(1-q) \tilde{\beta} / \beta_{c}^{(1)}}\right)^{D+1} \frac{\zeta(D+1)}{\zeta(D)} \frac{\phi\left(D, \frac{2-q}{1-q}+D,\langle\hat{N}\rangle_{1}\left(\frac{1+(1-q) \tilde{\beta} U_{q}}{(1-q) \tilde{\beta} / \beta_{c}^{(1)}}\right)^{D} \frac{\zeta(D+1)}{\zeta(D)}\right)}{\phi\left(D, \frac{1}{1-q},\langle\hat{N}\rangle_{1}\left(\frac{1+(1-q) \tilde{\beta} U_{q}}{(1-q) \tilde{\beta} / \beta_{c}^{(1)}}\right)^{D} \frac{\zeta(D+1)}{\zeta(D)}\right)},
\end{gathered}
$$

where $\phi(\alpha, \gamma ; z)=\sum_{k=0}^{\infty} z^{k} /(k ! \Gamma(\alpha k+\gamma))$ with $\alpha, \gamma>$ $0 \tilde{\beta}=\beta / \operatorname{Tr} \rho^{q},\langle\hat{N}\rangle_{1} \propto\left(1 / \beta_{c}^{(1)}\right)^{D}$ and $\beta_{c}^{(1)}=1 / T_{1}$ where $T_{1}$ is the critical temperature. More details about the calculation above can be found in [20], where the free particle case has been analyzed.

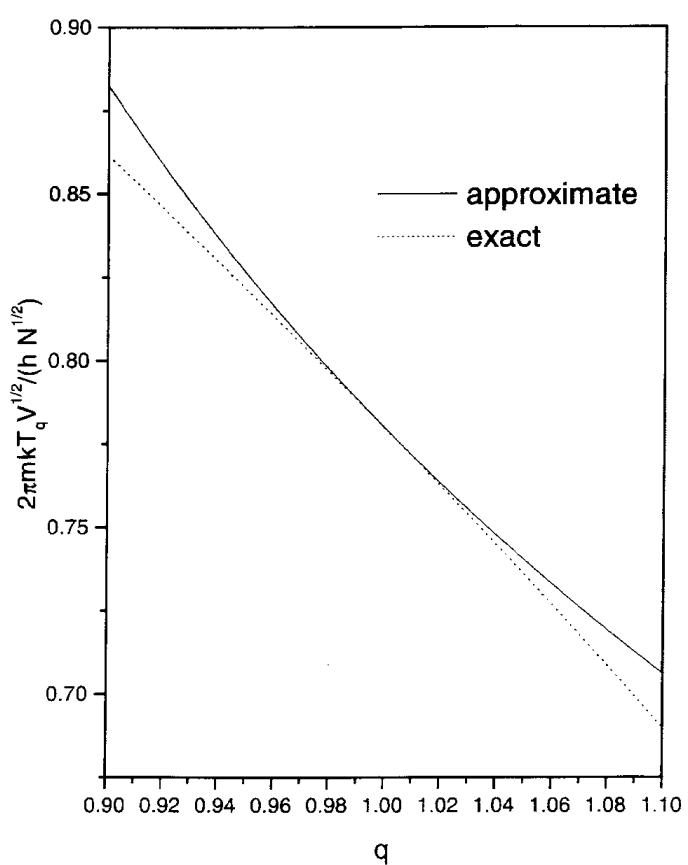

Figure.1 We show the critical temperature versus the nonextensive parameter $q$ for the homogeneous gas, considering $D=4$. It should be noted that we also extend the approximate result for $q<1$

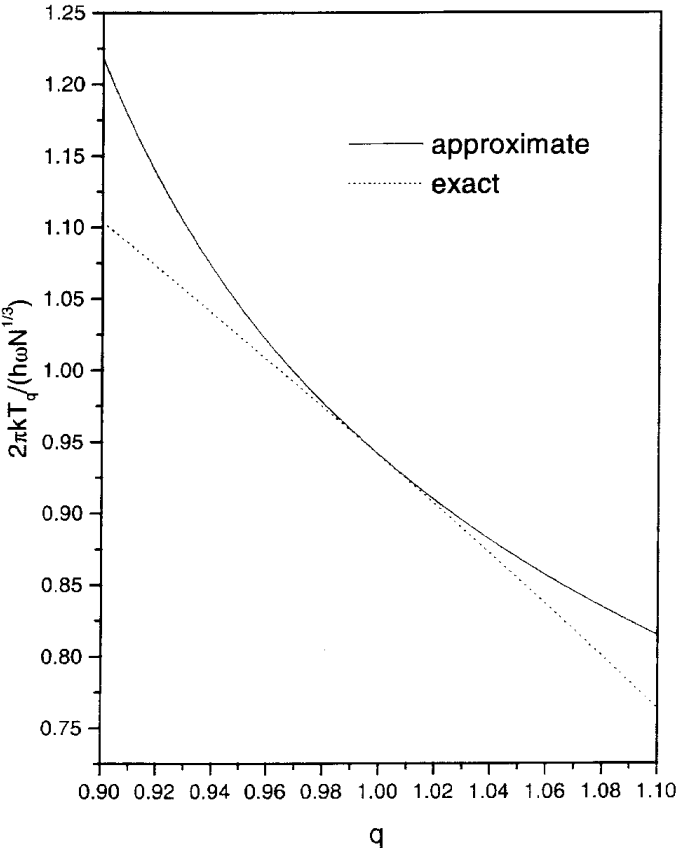

Figure. 2 Plots of critical temperature versus the nonextensive parameter $q$ for a gas in a harmonic trap. Here, the three dimension $(D=3)$ case is considered.

In summary, we have obtained the exact standard BEC formula by using the generalized Bose-Einstein distribution, within the dilute gas assumption, in Tsallis statistics. The comparison between our results and those obtained in [12] shows that the approximation Eq.(2) is not accurate for most situations examined here. Thus, the results obtained for the Bose-Einstein 
condensation $[12,14,15]$ and among others, in the light of our results, should be reanalysed. The role of the interactions in the BEC $[21,22]$ in Tsallis statistics will be addressed in an other opportunity. Furthermore, we have also found the expressions for the BEC within the normalized constraints, in order to promote a possible analysis involving Lagrange parameter, temperature, constraints and experimental data. Finally, we believe that this work can be useful in the analyses of future applications which involve Tsallis statistics and BEC, within the dilute gas assumption.

EKL thanks CNPq and PRONEX (Brazilian agencies) for financial support.

\section{References}

[1] C. Tsallis, J. Stat. Phys. 52, 479 (1988); see also Chaos, Solitons and Fractals 6, 539 (1995); E. M. F. Curado and C. Tsallis, J. Phys. A 24, L69 (1991); Errata: 24, 3187 (1991); 25, 1019 (1992).

[2] C. Tsallis, R. S. Mendes and A. R. Plastino, Physica A 261, 534 (1998).

[3] M.Buiatti, P. Grigolini, and A. Montagnini, Phys. Rev. Lett. 82, 3383 (1999); see also references therein.

[4] B. M. Boghosian, Phys. Rev. E 53, 4754 (1996).

[5] A. R. Plastino and A. Plastino, Phys. Lett. A 174, 384 (1993); J. J. Aly, Proceedings of N-Body Problems and Gravitational Dynamics, Aussois, France ed F. Combes and E, Athanassoula Publications de l'Observatoire de Paris, Paris, 1993, p. 19; V. H. Hamity and D. E. Barraco, Phys. Rev. Lett. 76, 4664 (1996).

[6] I. Koponen, Phys. Rev. E 55, 7759 (1997).

[7] P. Jund, S. G. Kim and C. Tsallis, Phys. Rev. B 52, 50 (1995).

[8] http://tsallis.cat.cbpf.br/biblio.htm.
[9] A. K. Rajagopal, R. S. Mendes and E. K. Lenzi, Phys. Rev.Lett. 80, 3907 (1998); E. K. Lenzi, R. S. Mendes and A. K. Rajagopal, Phys. Rev. E 59, 1397 (1999).

[10] R. A. Treumann, Europhys. Lett. 48, 8 (1999).

[11] D. F. Torres, H. Vucetchi, A. Plastino, Phys. Rev. Lett. 79, 1588 (1997).

[12] L. Salasnich, Int. J. Mod. Phys. B 14, 405 (2000).

[13] F. Buyukkilic, D. Demirhan and A. Gulec, Phys. Lett. A 197, 209 (1995).

[14] U. Tirnakli, F. Buyukkilic and D. Dermirhan, Physica A 240, 657 (1997); S. F. Ozeren et al., Eur. Phys. J. B 2, 101 (1998); U. Tirnakli, F. Buyukkilic and D. Dermirhan, Phys. Lett. A 245, 62 (1998); U. Tirnakli and D. F. Torres, Physica A 268 , 225 (1999).

[15] D. F. Torres and U. Tirnakli, Physica A 261, 499 (1998).

[16] I.S. Oliveira, Eur. Phys. J. B 14, 43 (2000).

[17] S. Curilef, A. R. R. Papa, Int. J. Mod. Phys. B 11, 2303 (1997).

[18] D. S. Grewall, Phys. Rev. B 18, 2127 (1978); Erratum: Phys. Rev. E 21, 1329 (1978).

[19] K. Sekar, G. Kuri, P.V. Satyam, B. Sandaravel, D.P. Mahapatra and B.N. Dev, Sol. St. Com. 96, 871 (1995); B.X. Liu, Nucl. Instr. Met. B: Beam Int. Mat. Atoms 59, 475 (1991); C.W. Lung, Frac. Strengh Sol., parts 1\&2 145 (1998); S.Z. Zhang, Zeitschrift, fur Angewandte Mathematik und Mechanik 70, 530 (1990).

[20] E. K. Lenzi, R. S. Mendes and A. K. Rajagopal, Physica A 286, 503 (2000).

[21] A. L. Fetter and, J. D. Walecka, Quantum Theory of Many Particle Systems, ( Mcgraw-Hill Publishing Co., New York, 1971).

[22] A. S. Parkins and D. F. Walls, Phys. Reports 303, 1 (1998); F. Dalfovo et. al., Rev. Mod. Phys. 71, 463 (1999). 